\title{
On the stability of the Hartmann layer
}

R. J. Lingwood, and T. Alboussière

Citation: Physics of Fluids 11, 2058 (1999); doi: 10.1063/1.870068

View online: https://doi.org/10.1063/1.870068

View Table of Contents: http://aip.scitation.org/toc/phf/11/8

Published by the American Institute of Physics

\section{Articles you may be interested in}

A model for the turbulent Hartmann layer

Physics of Fluids 12, 1535 (2000); 10.1063/1.870402

Study of instabilities and quasi-two-dimensional turbulence in volumetrically heated magnetohydrodynamic flows in a vertical rectangular duct

Physics of Fluids 25, 024102 (2013); 10.1063/1.4791605

Three-dimensional numerical simulations of magnetohydrodynamic flow around a confined circular cylinder under low, moderate, and strong magnetic fields

Physics of Fluids 25, 074102 (2013); 10.1063/1.4811398

On the measurement of the Hartmann layer thickness in a high magnetic field

Physics of Fluids 16, 3243 (2004); 10.1063/1.1768871

Amplification of small perturbations in a Hartmann layer

Physics of Fluids 14, 1458 (2002); 10.1063/1.1456512

Stability of plane Hartmann flow subject to a transverse magnetic field

The Physics of Fluids 16, 1848 (1973); 10.1063/1.1694224

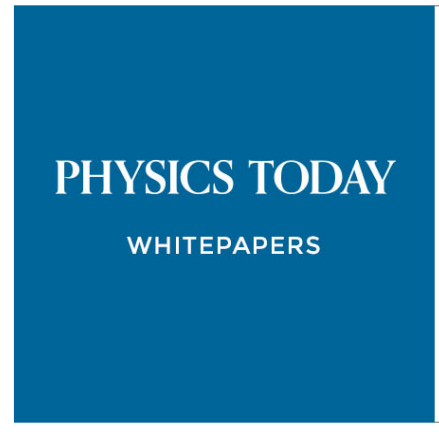

ADVANCED LIGHT CURE ADHESIVES

Take a closer look at what these environmentally friendly adhesive systems can do

\section{READ NOW}

PRESENTED BY (8) MASTERBOND० 


\title{
On the stability of the Hartmann layer
}

\author{
R. J. Lingwood ${ }^{\text {a) }}$ and T. Alboussière \\ Department of Engineering, University of Cambridge, Trumpington Street, \\ Cambridge CB2 1PZ, United Kingdom
}

(Received 22 December 1998; accepted 23 April 1999)

\begin{abstract}
In this paper we are concerned with the theoretical stability of the laminar Hartmann layer, which forms at the boundary of any electrically conducting fluid flow under a steady magnetic field at high Hartmann number. We perform both linear and energetic stability analyses to investigate the stability of the Hartmann layer to both infinitesimal and finite perturbations. We find that there is more than three orders of magnitude between the critical Reynolds numbers from these two analyses. Our interest is motivated by experimental results on the laminar-turbulent transition of ducted magnetohydrodynamics flows. Importantly, all existing experiments have considered the laminarization of a turbulent flow, rather than transition to turbulence. The fact that experiments have considered laminarization, rather than transition, implies that the threshold value of the Reynolds number for stability of the Hartmann layer to finite-amplitude, rather than infinitesimal, disturbances is in better agreement with the experimental threshold values. In fact, the critical Reynolds number for linear instability of the Hartmann layer is more than two orders of magnitude larger than experimentally observed threshold values. It seems that this large discrepancy has led to the belief that stability or instability of the Hartmann layer has no bearing on whether the flow is laminar or turbulent. In this paper, we give support to Lock's hypothesis [Proc. R. Soc. London, Ser. A 233, 105 (1955)] that "transition" is due to the stability characteristics of the Hartmann layer with respect to large-amplitude disturbances. (C) 1999 American Institute of Physics.
\end{abstract}

[S1070-6631(99)03708-3]

\section{INTRODUCTION}

The Hartmann layer is a fundamental element of magnetohydrodynamics (MHD). It develops along any boundary in an electrically conducting fluid where the magnetic field is not tangential to the boundary and it is where most of the shear stress is concentrated. More than being simply a hydrodynamical boundary layer, it provides a path for electrical currents that close within the core of the flow; the Hartmann layer thus controls the whole flow. The stability and the state (i.e., whether laminar or turbulent) of the Hartmann layer are important for two reasons. First, as with a classical boundary layer, the transfer of heat or mass through the layer depends fundamentally on its state. Second, if a laminar Hartmann layer is destabilized, the global electric circulation is affected and the flow may completely change in nature and intensity. In general, application of a magnetic field to a boundary layer has two effects on the stability of the layer. First, the magnetic field acts to accelerate the damping of perturbations through Joule dissipation and, second, the field deforms the laminar velocity profile and hence changes the hydrodynamical stability characteristics of the boundary layer.

Examples of applications in which the stability of the Hartmann layers may be of importance are numerous. For instance, in the field of crystal growth, steady magnetic fields are used essentially to stabilize the flow and, to a lesser extent, to control the dopant distribution in the final product.

a)Electronic mail: rj12@eng.cam.ac.uk
The stability of Hartmann layers must be ensured. In fact, this stability requirement could be a criterion for determining the minimum magnetic field intensity to apply, although as yet no direct evidence of this potential cause of instability has been shown. Metallurgy and, in particular, steel casting makes use of steady (or slowly sliding) magnetic fields. The nature of the flow is not well known, but the velocities and dimensions are large, of the order of a meter per second and a meter, respectively. In such cases, the Hartmann-layer stability may be of primary importance in determining the global damping effect of the magnetic field. In fusion-reactor projects, a so-called liquid-metal blanket surrounds the plasma and is subjected to an intense magnetic field of several teslas. The natural convection, which develops due to the large heat flux received, produces large velocities and therefore the stability of the Hartmann layer should be investigated. Finally, the case of MHD-generated twodimensional turbulence is linked to the state of the Hartmann layer. It is generally assumed that the layer is laminar and therefore simply provides a "frictional" linear damping force on the two-dimensional core turbulence. If, however, the Hartmann layer becomes unstable, this linear term should be replaced by another model.

The concept of the Hartmann layer was introduced by Hartmann and Lazarus. ${ }^{1}$ Its thickness $\delta^{*}$ depends only on the fluid's properties and the magnetic field intensity $B^{*}$. If $H^{*}$ is a typical length scale of the flow, $\delta^{*} / H^{*} \sim \mathrm{Ha}^{-1}$, where the Hartmann number is given by $\mathrm{Ha}$ $=\left(\sigma^{*} /\left(\rho^{*} \nu^{*}\right)\right)^{1 / 2} B^{*} H^{*}$. Here the asterisks denote dimen- 
sional quantities, $\rho^{*}$ is the fluid density, $\nu^{*}$ is the kinematic viscosity, and $\sigma^{*}$ is the electrical conductivity. When a typical velocity $U^{*}$ is considered in a cavity, a Reynolds number can be formed $\operatorname{Re}=U^{*} H^{*} / \nu^{*}$. As recognized first by Lundquist, ${ }^{2}$ the ratio $\mathrm{Re} / \mathrm{Ha}$ is the Reynolds number based on the Hartmann-layer thickness and therefore should be the governing parameter for the stability of the layer. Lock ${ }^{3}$ provided the first linear stability analysis of the Hartmann layer. In his study, he neglected the Lorentz force acting on the disturbances and found that the critical ratio $\mathrm{Re} / \mathrm{Ha}$ seems to converge toward $5.0 \times 10^{4}$ in the limit of high Hartmann number. Roberts ${ }^{4}$ provides an approximate solution for the critical Reynolds number (46 200). More recently, Takashima ${ }^{5,6}$ performed a linear stability analysis for Poiseuille and Couette flow under a transverse magnetic field, taking into account the Lorentz force on the disturbances and finite values of the magnetic Prandtl number, for Hartmann numbers up to 200 . He found in both cases that the critical ratio Re/Ha converges toward 48311.016 for high Hartmann numbers at low magnetic Prandtl number and that increasing the magnetic Prandtl number is slightly destabilizing. The common feature of all these stability studies is that the magnetic field is strictly perpendicular to the boundary and that the analysis has been performed for a more "global" flow than an isolated Hartmann layer; namely, a Poiseuille or Couette flow.

The experimental results reported in the literature are not explicitly dedicated to the stability of the Hartmann layer. Several authors (e.g., Hartmann and Lazarus, ${ }^{1}$ Murgatroyd, ${ }^{7}$ Lykoudis, ${ }^{8}$ and Branover ${ }^{9}$ ) determine whether a duct flow is laminar or turbulent in the presence of a transverse magnetic field. The flow is turbulent before entering the gap between the magnet poles and what is really recorded is the smallest value of transverse magnetic field necessary to laminarize the flow. (In general the difference between transition and laminarization has not been emphasized in the literature; with a few exceptions, laminarization is rather loosely called transition without qualification.) The value reported by all experimentalists for this laminarization corresponds to a ratio $150<\mathrm{Re} / \mathrm{Ha}<250$ for sufficiently large Hartmann numbers and electrically insulating walls. This result is extremely robust, in the sense that it is valid for rectangular cross sections of any aspect ratio and also for circular pipes. Despite the fact that experiments show that $\mathrm{Re} / \mathrm{Ha}$ is a consistent indicator of laminarization, it is not generally accepted that instability of the Hartmann layers controls the process. At the end of his paper, Lock ${ }^{3}$ expresses his concerns about the discrepancy between his critical Reynolds number, derived from a linear stability analysis, and experimental evidence and argues that instability to finite-amplitude disturbances may resolve the difference.

The structure of the paper is as follows. In Sec. II we define the configuration. Section III is devoted to the linear stability analysis, including investigation of the effect of inclining the magnetic field away from the wall-normal direction. In Sec IV the energetic stability of the Hartmann layer is examined for the first time. These results are followed by a discussion and conclusions in Sec. V.

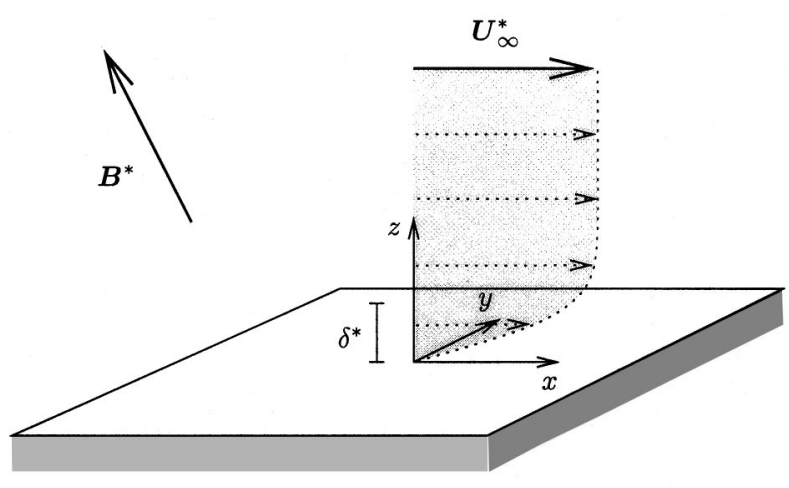

FIG. 1. The studied configuration.

\section{THE BASE FLOW}

Under the assumption of a small magnetic Reynolds number, the dimensional Navier-Stokes and continuity equations for an electrically conducting fluid with an imposed magnetic field vector $\mathbf{B}^{*}$ are

$$
\begin{aligned}
\frac{\partial \mathbf{u}^{*}}{\partial t^{*}}+\left(\mathbf{u}^{*} \cdot \boldsymbol{\nabla}\right) \mathbf{u}^{*}= & -\frac{1}{\rho^{*}} \boldsymbol{\nabla} p^{*}+\frac{1}{\rho^{*}}\left(\mathbf{j}^{*} \wedge \mathbf{B}^{*}\right) \\
& +\nu^{*} \nabla^{2} \mathbf{u}^{*}, \quad \boldsymbol{\nabla} \cdot \mathbf{u}^{*}=0,
\end{aligned}
$$

where the asterisks denote dimensional quantities, $t^{*}$ is time, $\mathbf{u}^{*}$ is the velocity vector field, $p^{*}$ is the pressure field, and $\mathbf{j}^{*}$ is the current density vector field. Ohm's law for a moving electrically conducting medium is given by

$$
\mathbf{j}^{*}=\sigma^{*}\left(-\boldsymbol{\nabla} \varphi^{*}+\mathbf{u}^{*} \wedge \mathbf{B}^{*}\right), \quad \boldsymbol{\nabla} \cdot \mathbf{j}^{*}=0,
$$

where $\varphi^{*}$ is the electric potential field.

The configuration considered is a Hartmann layer, isolated from a global MHD flow (see Fig. 1). The boundary is assumed to be flat at the scale of the Hartmann-layer thickness and the tangential free-stream velocity vector field $\mathbf{U}_{\infty}^{*}$ is taken to be uniform. The uniform magnetic field $\mathbf{B}^{*}$ is not parallel to the boundary.

The nondimensionalizing velocity scale is $U_{\infty}^{*}$, which is the free-stream velocity, and the length scale is $\delta^{*}$ $=\left(\nu^{*} \rho^{*} / \sigma^{*}\right)^{1 / 2} / B_{z}^{*}$, which is the boundary-layer length scale and where $B_{z}^{*}$ is the magnitude of the wall-normal component of $\mathbf{B}^{*}$. Time, pressure, magnetic field, and current density scales are $\delta^{*} / U_{\infty}^{*}, \rho^{*} U_{\infty}^{* 2}, B_{z}^{*}$, and $\sigma^{*} U_{\infty}^{*} B_{z}^{*}$, respectively. The Reynolds number $R=U_{\infty}^{*} \delta^{*} / \nu^{*}$ $=\left(\sigma^{*} \nu^{*} / \rho^{*}\right)^{-1 / 2} U_{\infty}^{*} / B_{z}^{*}(\mathrm{Re} / \mathrm{Ha}$ in the notation of Sec. I) is the single dimensionless parameter for the Hartmann layer. One may think that the Stuart number (also called the interaction parameter) $N=\sigma^{*} B_{z}^{* 2} \delta^{*} /\left(\rho^{*} U_{\infty}^{*}\right)$ is also an independent parameter, but it can be shown that $N=1 / R$. The problem is formulated in Cartesian coordinates with $x$ denoting the streamwise direction, $y$ denoting the spanwise direction, and $z$ denoting the wall-normal direction.

The dimensionless steady solutions for the velocity and current density vector fields are given by

$$
\begin{aligned}
& \mathbf{U}=[U(z), 0,0]=\left[1-e^{-z}, 0,0\right], \\
& \mathbf{J}=[0, J(z), 0]=\left[0, e^{-z}, 0\right],
\end{aligned}
$$






FIG. 2. The base flow: $-U(z) ;---J(z)$.

and shown in Fig. 2. This ideal expression for the Hartmann layer solution is appropriate for the study of the stability of any boundary layer in the limit of high Hartmann number, provided the magnetic field is not parallel to the wall. In reality the free-stream velocity may not be strictly uniform, but can be considered as such over a much larger length scale than the Hartmann-layer thickness. Also, instead of vanishing in the core, the value of the current density may converge toward a finite value, in which case a pressure gradient would balance the Lorentz force in the core. This finite value and the associated pressure gradient would not affect the stability analysis and can be subtracted to recover expression (2.3).

\section{LINEAR STABILITY ANALYSIS}

\section{A. Formulation}

In this section we consider the effect of superimposing an infinitesimal disturbance on the steady fields. The instantaneous velocities, pressure, current density, and electric potential are given by

$$
\begin{aligned}
& u(x, y, z, t)=U(z)+\widetilde{u}(x, y, z, t), \\
& v(x, y, z, t)=\widetilde{v}(x, y, z, t), \\
& w(x, y, z, t)=\widetilde{w}(x, y, z, t), \\
& p(x, y, z, t)=P(z)+\widetilde{p}(x, y, z, t), \\
& \mathbf{j}(x, y, z, t)=\mathbf{J}(z)+\widetilde{\mathbf{j}}(x, y, z, t), \\
& \varphi(x, y, z, t)=\Phi(z)+\widetilde{\varphi}(x, y, z, t),
\end{aligned}
$$

where $\tilde{u}, \widetilde{v}, \widetilde{w}, \widetilde{p}, \widetilde{\mathbf{j}}$, and $\widetilde{\varphi}$ are the small perturbation quantities. The perturbation quantities take the following normalmode form:

$$
\begin{aligned}
{[\tilde{u}, \widetilde{v}, \widetilde{w}, \widetilde{p}, \tilde{j}, \widetilde{\varphi}]^{T}=} & {[\hat{u}(z), \hat{v}(z), \hat{w}(z), \hat{p}(z), \hat{\jmath}(z), \hat{\varphi}(z)]^{T} } \\
& \times \exp \left[i\left(k_{x} x+k_{y} y-\omega t\right)\right],
\end{aligned}
$$

because the base flow is invariant in $x, y$, and $t$. The dimensionless forms of (2.1) and (2.2) are linearized with respect to the perturbation quantities, which for each solution set $\left(k_{x}, k_{y}, \omega, R\right)$ results in an eighth-order system of ordinarydifferential equations in $(\hat{u}, \hat{v}, \hat{w}, \hat{p}, \hat{\varphi})$. Here, the full dependence of $\hat{u}(z)$ for example, is given by $\hat{u}\left(z, k_{x}, k_{y}, \omega, R\right)$ and $\hat{u}$ is the spectral representation of the $x$ velocity, $k_{x}$ and $k_{y}$ are the streamwise and spanwise wave numbers, respectively, and $\omega$ is the frequency of the perturbation. The real part of (3.2) is taken to obtain physical quantities. The perturbation equations can be written as a set of eight first-order ordinary differential equations in the following transformed variables:

$$
\begin{aligned}
& \phi_{1}\left(z, k_{x}, k_{y}, \omega, R\right)=k_{z} \hat{u}+k_{y} \hat{v}, \\
& \phi_{2}\left(z, k_{x}, k_{y}, \omega, R\right)=k_{x} \hat{u}^{\prime}+k_{y} \hat{v}^{\prime}, \\
& \phi_{3}\left(z, k_{x}, k_{y}, \omega, R\right)=\hat{w}, \quad \phi_{4}\left(z, k_{x}, k_{y}, \omega, R\right)=\hat{p}, \\
& \phi_{5}\left(z, k_{x}, k_{y}, \omega, R\right)=k_{x} \hat{v}-k_{y} \hat{u}, \\
& \phi_{6}\left(z, k_{x}, k_{y}, \omega, R\right)=k_{x} \hat{v}^{\prime}-k_{y} \hat{u}^{\prime}, \\
& \phi_{7}\left(z, k_{x}, k_{y}, \omega, R\right)=\hat{\varphi}, \quad \phi_{8}\left(z, k_{x}, k_{y}, \omega, R\right)=\hat{\varphi}^{\prime},
\end{aligned}
$$

where the primes denote differentiation with respect to $z$. Let us first perform the analysis for a magnetic field purely perpendicular to the wall: $\mathbf{B}=\mathbf{e}_{z}$. In the transformed variables, the perturbation equations are

$$
\left(\begin{array}{c}
\phi_{1}^{\prime} \\
\phi_{2}^{\prime} \\
\phi_{3}^{\prime} \\
\phi_{4}^{\prime} \\
\phi_{5}^{\prime} \\
\phi_{6}^{\prime} \\
\phi_{7}^{\prime} \\
\phi_{8}^{\prime}
\end{array}\right)=\left(\begin{array}{cccccccc}
0 & 1 & 0 & 0 & 0 & 0 & 0 & 0 \\
A+1 & 0 & R k_{x} U^{\prime} & i R k^{2} & 0 & 0 & 0 & 0 \\
-i & 0 & 0 & 0 & 0 & 0 & 0 & 0 \\
0 & -i / R & -A / R & 0 & 0 & 0 & 0 & 0 \\
0 & 0 & 0 & 0 & 0 & 1 & 0 & 0 \\
0 & 0 & -R k_{y} U^{\prime} & 0 & A+1 & 0 & -i k^{2} & 0 \\
0 & 0 & 0 & 0 & 0 & 0 & 0 & 1 \\
0 & 0 & 0 & 0 & i & 0 & k^{2} & 0
\end{array}\right)\left(\begin{array}{c}
\phi_{1} \\
\phi_{2} \\
\phi_{3} \\
\phi_{4} \\
\phi_{5} \\
\phi_{6} \\
\phi_{7} \\
\phi_{8}
\end{array}\right),
$$


where $k^{2}=k_{x}^{2}+k_{y}^{2}$ and $A=i R\left(k_{x} U-\omega\right)+k^{2}$.

This system has eight independent solutions $\phi_{i}^{j}$ $\left(z, k_{x}, k_{y}, \omega, R\right)(i=j=1,2,3, \ldots, 8)$, where the subscript $i$ indicates one of the eight transformed variables and the superscript $j$ indicates one of the eight solutions. These solutions cannot be found analytically because the $8 \times 8$ matrix depends on $z$ through the base flow $U$, but the perturbation equations in the limit $z \rightarrow \infty$, where $U \rightarrow 1$ and $U^{\prime} \rightarrow 0$ have exact closed-form solutions that are exponential in form:

$\phi_{i}^{j}\left(z \rightarrow \infty, k_{x}, k_{y}, \omega, R\right)=c_{i}^{j} e^{\kappa_{j} z}, \quad i=j=1,2,3, \ldots, 8$,

where $c_{i}^{j}$ represent constant coefficients that are the components of the eigenvectors of the $8 \times 8$ matrix in (3.4) in the limit $z \rightarrow \infty$. The values of $\kappa_{j}$ are the eigenvalues of the same matrix in the limit $z \rightarrow \infty$ and are given by

$$
\begin{aligned}
\kappa_{1,2}=\kappa_{5,6}= & \mp(1 / 2)\left(2+4 k^{2}+2 i R\left(k_{x}-\omega\right)\right. \\
& +2\left(1+4 k^{2}+2 i R\left(k_{x}-\omega\right)\right. \\
& \left.\left.+2 R^{2} k_{x} \omega-R^{2} \omega^{2}-R^{2} k_{z}^{2}\right)^{1 / 2}\right)^{1 / 2}, \\
\kappa_{3,4}=\kappa_{7,8}= & \mp(1 / 2)\left(2+4 k^{2}+2 i R\left(k_{x}-\omega\right)\right. \\
& -2\left(1+k^{2}+2 i R\left(k_{x}-\omega\right)\right. \\
& \left.\left.+2 R^{2} k_{x} \omega-R^{2} \omega^{2}-R^{2} k_{x}^{2}\right)^{1 / 2}\right)^{1 / 2} .
\end{aligned}
$$

The eight fundamental solutions are obtained by numerical integration of (3.4) through the Hartmann layer down to the wall starting from the eight eigenvectors valid at sufficient large distance from the wall. The solution to (3.4) is formed by summing the eight fundamental solutions, with appropriate weightings, in such a way that the boundary conditions are satisfied. Components of the final solution vector are thus represented by

$$
\begin{aligned}
\phi_{i}\left(z, k_{x}, k_{y}, \omega, R\right)= & \sum_{j=1}^{8} C_{j}\left(k_{x}, k_{y}, \omega, R\right) \phi_{i}^{j}\left(z, k_{x}, k_{y}, \omega, R\right), \\
& i=1,2,3, \ldots, 8,
\end{aligned}
$$

where $C_{j}\left(k_{x}, k_{y}, \omega, R\right)$, which is constant with respect to $z$, is the weighting coefficient of the $j$ th solution vector. The boundary conditions at $z \rightarrow \infty$ are that all perturbations decay, which implies that $C_{2}=C_{4}=C_{6}=C_{8}=0$. The boundary conditions at $z=0$ are that $\hat{u}(0)=\hat{v}(0)=\hat{w}=0$ and that either $\varphi(0)=0$ or $\varphi^{\prime}(0)=0$, depending on whether the boundary is assumed to be perfectly conducting or perfectly insulating (we considered only these extreme cases). These homogeneous wall boundary conditions determine the values of $C_{1}$, $C_{3}, C_{5}$, and $C_{7}$, which can be found from

$$
\begin{aligned}
\left(\begin{array}{c}
\phi_{1}(0) \\
\phi_{3}(0) \\
\phi_{5}(0) \\
\phi_{7,8}(0)
\end{array}\right) & =\left(\begin{array}{cccc}
\phi_{1}^{1}(0) & \phi_{1}^{3}(0) & \phi_{1}^{5}(0) & \phi_{1}^{7}(0) \\
\phi_{3}^{1}(0) & \phi_{3}^{3}(0) & \phi_{3}^{5}(0) & \phi_{3}^{7}(0) \\
\phi_{5}^{1}(0) & \phi_{5}^{3}(0) & \phi_{5}^{5}(0) & \phi_{5}^{7}(0) \\
\phi_{7,8}^{1}(0) & \phi_{7,8}^{3}(0) & \phi_{7,8}^{5}(0) & \phi_{7,8}^{7}(0)
\end{array}\right) \\
& \times\left(\begin{array}{c}
C_{1} \\
C_{3} \\
C_{5} \\
C_{7}
\end{array}\right)=\left(\begin{array}{c}
0 \\
0 \\
0 \\
0
\end{array}\right)
\end{aligned}
$$

where the subscript 7 or 8 is chosen depending on whether the boundary is conducting or insulating, and the dependence on $k_{x}, k_{y}, \omega$, and $R$ has been omitted from the list of variables. Letting the partial Wronskian-determinant of the 4 $\times 4$ matrix in (3.8) - be $\Delta\left(k_{x}, k_{y}, \omega, R\right)$, we have nontrivial solutions to the homogeneous problem only when $\Delta\left(k_{x}, k_{y}, \omega, R\right)=0$; this is usually referred to as the dispersion relation. Further, combinations of $k_{x}, k_{y}, \omega$, and $R$ that satisfy the dispersion relation define eigenvalues $\omega$ and the resulting solutions of (3.7) are the corresponding eigenfunctions.

When the dispersion relation is satisfied, the $4 \times 4$ matrix in (3.8) is singular and $C_{1,3,5,7}$ can be determined using singular-value decomposition, ${ }^{10}$ which then allows the eigenfunctions to be calculated. With any three of the dependent variables given, the solution of the dispersion relation allows the fourth to be determined. We have a two-point boundaryvalue problem and we used a shooting method of solution, which involves choosing values for the fourth unknown dependent variable at the start point and integrating the perturbation equations to the wall, which in general results in a nonzero value of $\Delta\left(k_{x}, k_{y}, \omega, R\right)$. A Newton-Raphson linear search procedure was used to find the starting values of the initially guessed variable that zero $\Delta\left(k_{x}, k_{y}, \omega, R\right)$ to within a predetermined tolerance. We used a fixed-step-size fourthorder Runge-Kutta integration scheme to integrate through the boundary layer to the wall, starting from the exact solutions of the perturbation equations at $z \rightarrow \infty$, given by (3.5). We took $z=5$ to be sufficiently far from the wall to be a good approximation for $z \rightarrow \infty$. Gram-Schmidt orthonormalization was used to cope with the stiffness of the perturbation equations (thus, in conjunction with shooting, we use a socalled "parallel-shooting", technique). The orthonormalization procedure replaces the original vectors with an orthonormal set leaving discontinuities in the vectors at each point of orthonormalization, which means that the solution vectors must be reconstructed before they can be combined to give the eigenfunctions. A standard method of reconstruction was used; see Wazzan, Okamura, and Smith. ${ }^{11}$

The perturbation equations can also be written as two fourth-order equations,

$$
\begin{aligned}
&\left(k_{x} U-\omega\right)\left(\hat{w}^{\prime \prime}-k^{2} \hat{w}\right)-k_{x} U^{\prime \prime} \hat{w} \\
&+ \frac{i}{R}\left(\hat{w}^{\prime \prime \prime \prime}-\left(2 k^{2}+1\right) \hat{w}^{\prime \prime}+k^{4} \hat{w}\right)=0, \\
&\left(k_{x} U-\omega\right)\left(\hat{\varphi}^{\prime \prime}-k^{2} \hat{\varphi}\right)-k_{y} U^{\prime} \hat{w} \\
&+\frac{i}{R}\left(\hat{\varphi}^{\prime \prime \prime}-\left(2 k^{2}+1\right) \hat{\varphi}^{\prime \prime}+k^{4} \hat{\varphi}\right)=0,
\end{aligned}
$$

which are, respectively, the familiar Orr-Sommerfeld and Squire's-mode equations appropriately modified to include the effects of an imposed steady (wall-normal) magnetic field. (Here we have given the equations in the primitive variables $\hat{\omega}$ and $\hat{\varphi}$, but we could equally have written them in terms of $\phi_{3}$ and $\phi_{7}$.) Note that (3.10) is coupled to (3.9) only if $k_{y} \neq 0$. This fact is also clear from (3.4), which is block diagonal if $k_{y}=0$ (as it also is in the limit of $z \rightarrow \infty$ ). In 




FIG. 3. Marginal curves for linear stability for both an insulating and conducting boundary and a purely wall-normal magnetic field.

fact, whether $k_{y}$ is zero or nonzero, solution of (3.9) alone [or, equivalently, the top left $4 \times 4$ block of (3.4) alone] will give the eigenvalues of the problem and it is only if the eigenfunctions are required that (3.10) [or, equivalently, the bottom right $4 \times 4$ block of (3.4)] must also be solved.

\section{B. Non-normal magnetic field}

The discussion so far has dealt entirely with the case where the imposed magnetic field is in the $z$ direction only. We have also considered the effects of inclination of the field in the $x$ and $y$ directions. Formulation of these problems is similar to that discussed in Sec. III A with appropriately modified forms of (3.4) and (3.6). The nondimensional imposed magnetic fields in the $x$ and $y$ directions are denoted by $B_{x}$ and $B_{y}$, respectively. Because the nondimensionalizing scale is $B_{z}^{*}, B_{x}$ and $B_{y}$ represent $\tan \theta_{x}$ and $\tan \theta_{y}$, respectively, where the angles are measured away from the positive $z$ direction. Due to our choice of nondimensionalizing scales, $B_{z}=1$ and therefore with increasing $B_{x}$ or $B_{y}$ the overall magnitude of the field increases but the base flow (2.3) is unchanged. Note that we have restricted the analyses by considering only the effects of $B_{x}$ or $B_{y}$ individually.

\section{Linear stability results}

We are interested in the critical Reynolds number for stability, i.e., the Reynolds number below which no infinitesimal perturbation grows, and, since Squire's theorem ${ }^{12}$ applies when the magnetic field is purely normal to the boundary, we have that the critical Reynolds number is given by a two-dimensional disturbance with $k_{y}=0$, i.e., by spanwise vortices. As can be seen from (3.4), the stability results are the same for conducting and insulating wall boundary conditions. Figure 3 shows the neutral curve for $k_{y}=0$, giving the critical Reynolds number for linear stability as $R$ $\approx 48250$. The linear stability of Poiseuille flow under a magnetic field was considered by Lock, ${ }^{3}$ but he neglected the
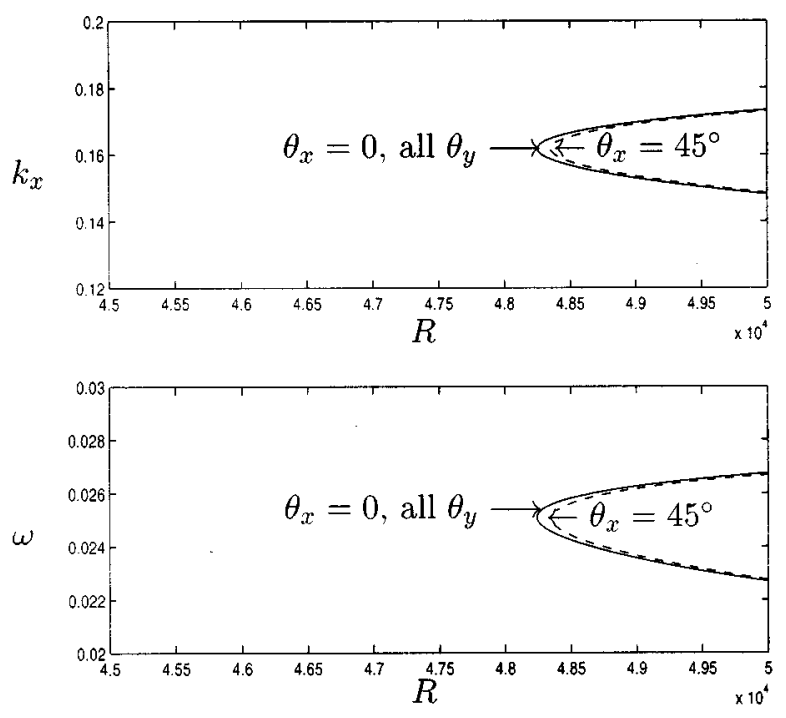

FIG. 4. Marginal curves for linear stability for both an insulating and conducting boundary and $k_{y}=0:-B_{x}=0$ and all $B_{y} ;-\cdots B_{x}=1$.

Lorentz force in the perturbation equations, which results in solution of the standard Orr-Sommerfeld equation (for which he used an approximate method) and therefore, with this mean velocity profile, corresponds equally to the asymptotic suction profile. (Note that Lock's value for the critical Reynolds number is 50000 .)

Inclination of the magnetic field in the $x$ direction is stabilizing to modes with $k_{y}=0$. Figure 4 shows the same marginal curve for linear stability as Fig. 3 but over a much reduced scale so that the small degree of stabilization produced by addition of $B_{x}=1$ can be seen. These curves apply equally to insulating and conducting boundaries, and the curve for $B_{x}=0$ also applies to any value of $B_{y}$; nonzero $B_{y}$ has no effect on the stability of modes with $k_{y}=0$. Furthermore, for $k_{y}=0$ the perturbations in electric potential $\hat{\varphi}$ and the spanwise velocity $v$ are both zero throughout the boundary layer; compare Fig. 5 with Fig. 6. Note that Fig. 6 is for an insulating boundary only; $\hat{\varphi}$ is nonzero at the wall, while $\hat{\varphi}^{\prime}$ (not plotted) is zero.

Although Squire's theorem is valid when the magnetic field is normal to the wall, it has not been proven when the field is inclined. Therefore, three-dimensional perturbations may lead to lower Reynolds numbers for linear stability when $B_{x} \neq 0$ or $B_{y} \neq 0$. However, there seems to be little benefit in analyzing this point in detail because the Reynolds numbers at which infinitesimal disturbances become unstable are so much larger than experimental observations for instability of the Hartmann layer. This discrepancy between the linear stability results and experimental observations led us to investigate the stability of the Hartmann layer to finite perturbations.

\section{ENERGETIC STABILITY ANALYSIS}

Linear stability is a relatively weak stability condition because it tells us nothing about the stability of finiteamplitude disturbances. To investigate the stability of any finite-amplitude perturbation the full nonlinear equations 


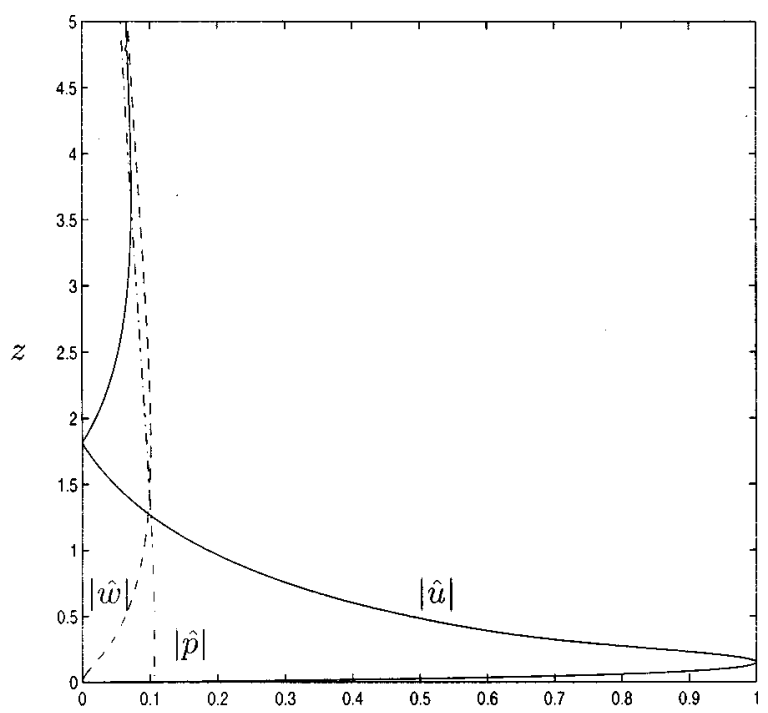

FIG. 5. Magnitude of all nonzero eigenfunctions normalized by the maximum in $\hat{u}$ for $R=50000$ on the upper branch of the marginal curve (see Figs. 3 and 4), for a wall-normal imposed magnetic field, for both an insulating and conducting boundary and for $k_{y}=0:-|\hat{u}| ;-\cdots|\hat{w}| ;-\cdot|\hat{p}|$.

must be used. The energetic stability method is first mentioned by $\mathrm{Orr}^{13}$ and a complete reference to this method can be found in Joseph. ${ }^{14}$

\section{A. Formulation}

The approach of Doering and Gibbon ${ }^{15}$ is followed and modified to account for the presence of a magnetic field. We define the condition for energetic stability to be that the kinetic energy of any disturbances must monotonically decay to zero. The energy decay is due to diffusive processes, momentum diffusion and magnetic diffusion, corresponding to viscous and Joule dissipation, respectively, while the mean flow acts as a reservoir of energy for the perturbations. So

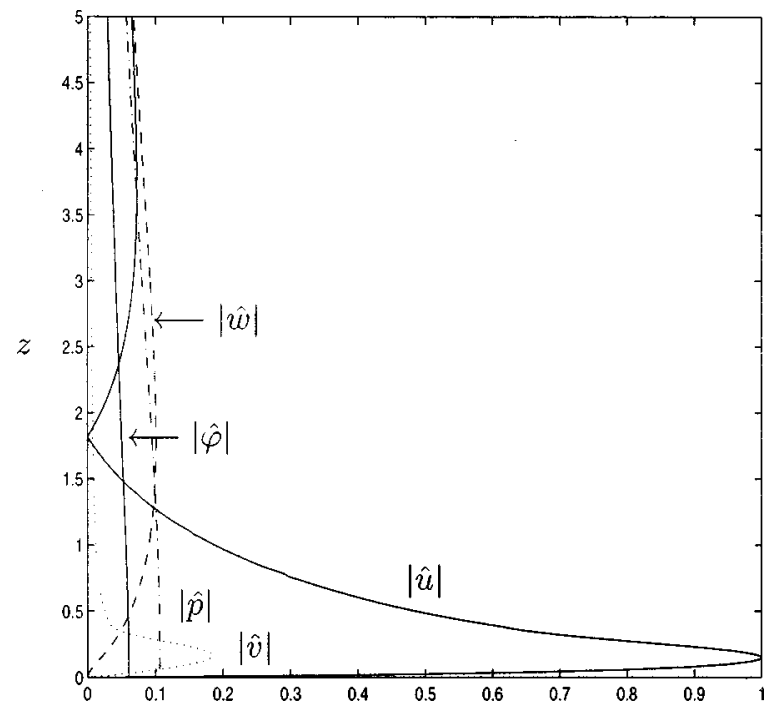

FIG. 6. Magnitude of all nonzero eigenfunctions normalized by the maximum in $\hat{u}$ for $R=50000$ on the upper branch of the marginal curve for a wall-normal imposed magnetic field, for an insulating boundary and for $k_{y}$ $= \pm 0.01:-|\hat{u}| ;-\cdots|\hat{w}| ;-\cdot|\hat{p}| ; \cdots|\hat{v}| ;-|\hat{\varphi}|$. the energetic-stability method described here is not to be confused with the inviscid energy-stability analysis, which is conservative and restricted to small-amplitude disturbances. In the latter method, under the constraints of the problem, the total energy of the base flow is found to be a local minimum among admissible flows, therefore preventing small disturbances from growing without limit. Taking (3.1) here to represent the addition of finite perturbations to the base state defined in Sec. II, then the perturbations satisfy

$$
\begin{aligned}
& \frac{\partial \widetilde{\mathbf{u}}}{\partial t}+(\widetilde{\mathbf{u}} \cdot \boldsymbol{\nabla}) \widetilde{\mathbf{u}}+(\widetilde{\mathbf{u}} \cdot \nabla) \mathbf{U}+(\mathbf{U} \cdot \nabla) \widetilde{\mathbf{u}} \\
& \quad=-\nabla \widetilde{p}+\frac{1}{R}\left(\widetilde{\mathbf{j}} \wedge \mathbf{B}+\nabla^{2} \widetilde{\mathbf{u}}\right), \quad \boldsymbol{\nabla} \cdot \widetilde{\mathbf{u}}=0, \\
& \widetilde{\mathbf{j}}=(-\nabla \widetilde{\varphi}+\widetilde{\mathbf{u}} \wedge \mathbf{B}), \quad \boldsymbol{\nabla} \cdot \widetilde{\mathbf{j}}=0 .
\end{aligned}
$$

Taking the scalar product of the momentum equation in (4.1) with $\widetilde{\mathbf{u}}$ and integrating over any finite volume with no net addition of energy, leads to the following energy equation:

$$
\frac{d}{d t}\|\widetilde{\mathbf{u}}\|_{2}^{2}=-\int_{\Omega} \widetilde{\mathbf{u}} \cdot(\boldsymbol{\nabla} \mathbf{U}) \cdot \widetilde{\mathbf{u}} d \mathbf{x}-\frac{1}{R}\|\widetilde{\mathbf{j}}\|_{2}^{2}-\frac{1}{R}\|\boldsymbol{\nabla} \widetilde{\mathbf{u}}\|_{2}^{2},
$$

where the $L^{2}$ norm is denoted by \|\|$_{2}$. It can be shown ${ }^{15}$ from Eq. (4.3) that the slowest possible decay rate of a perturbation is given by the following infimum over all divergence-free vector fields that satisfy the boundary conditions:

$$
\inf \left(\frac{(1 / R)\left(\|\nabla \widetilde{\mathbf{u}}\|_{2}^{2}+\|\widetilde{\mathbf{j}}\|_{2}^{2}\right)+\int_{\Omega} \widetilde{\mathbf{u}} \cdot(\nabla \mathbf{U}) \cdot \widetilde{\mathbf{u}} d \mathbf{x}}{\|\widetilde{\mathbf{u}}\|_{2}^{2}}\right),
$$

which can be calculated using variational calculus. The minimizing field is given by a field satisfying the Euler-Lagrange equations and therefore is a solution of the following eigenvalue problem:

$$
i \omega \widetilde{\mathbf{u}}=\widetilde{\mathbf{u}} \cdot \mathbf{D}-\frac{1}{R}\left(\widetilde{\mathbf{j}} \wedge \mathbf{B}+\nabla^{2} \widetilde{\mathbf{u}}\right)+\nabla \widetilde{p}, \quad \nabla \cdot \widetilde{\mathbf{u}}=0,
$$

where $\tilde{\mathbf{j}}$ is given by (4.2) and $D_{i j}=\left(\partial U_{i} / \partial U_{j}+\partial U_{j} / \partial x_{i}\right) / 2$ is the deformation tensor, which for $\mathbf{U}$ defined in (2.3) is given by $D_{13}=D_{31}=(\partial U(z) / \partial z) / 2$ with all other terms zero. The boundary conditions on the perturbations are the same as for the linear stability problem; see Sec. III A. Whereas the eigenvalue problem for the linear stability analysis is generally not self-adjoint, the nonlinear eigenvalue problem does result in a self-adjoint operator and therefore to a real spectrum of $i \omega$ or, equivalently, to an imaginary spectrum of $\omega$. If $\omega$ is negative then perturbations decay, and if $\omega$ is positive then they grow at least initially. We are interested in neutral values, i.e., $\omega=0$, and specifically in the critical Reynolds number below which no perturbation grows.

This eigenvalue problem is solved in an analogous way to the linear stability analysis. We recast (4.5) as eight firstorder ordinary differential equations in the transform variables of (3.3), which for $\mathbf{B}=\mathbf{e}_{\mathbf{z}}$ gives 


$$
\left(\begin{array}{c}
\phi_{1}^{\prime} \\
\phi_{2}^{\prime} \\
\phi_{3}^{\prime} \\
\phi_{4}^{\prime} \\
\phi_{5}^{\prime} \\
\phi_{6}^{\prime} \\
\phi_{7}^{\prime} \\
\phi_{8}^{\prime}
\end{array}\right)=\left(\begin{array}{cccccccc}
0 & 1 & 0 & 0 & 0 & 0 & 0 & 0 \\
1+k^{2} & 0 & R k_{x} U^{\prime} / 2 & i R k^{2} & 0 & 0 & 0 & 0 \\
-i & 0 & 0 & 0 & 0 & 0 & 0 & 0 \\
-k_{x} U^{\prime} /\left(2 k^{2}\right) & -i / R & -k^{2} / R & 0 & k_{y} U^{\prime} /\left(2 k^{2}\right) & 0 & 0 & 0 \\
0 & 0 & 0 & 0 & 0 & 1 & 0 & 0 \\
0 & 0 & -R k_{y} U^{\prime} / 2 & 0 & 1+k^{2} & 0 & -i k^{2} & 0 \\
0 & 0 & 0 & 0 & 0 & 0 & 0 & 1 \\
0 & 0 & 0 & 0 & i & 0 & k^{2} & 0
\end{array}\right)\left(\begin{array}{c}
\phi_{1} \\
\phi_{2} \\
\phi_{3} \\
\phi_{4} \\
\phi_{5} \\
\phi_{6} \\
\phi_{7} \\
\phi_{8}
\end{array}\right),
$$

where the notation is the same as in Sec. III, except that here the magnitudes of the perturbation quantities are no longer necessarily infinitesimal. This system is solved in the same way as (3.4). As before, the system has eight independent solutions $\phi_{i}^{j}\left(z, k_{x}, k_{y}, R\right)(i=j=1,2,3, \ldots, 8)$, where the subscript $i$ indicates one of the eight transformed variables and the superscript $j$ indicates one of the eight solutions, which have the following exact closed-form solutions in the limit $z \rightarrow \infty$ :

$$
\phi_{i}^{j}\left(z \rightarrow \infty, k_{x}, k_{y}, R\right)=c_{i}^{j} e^{\kappa_{j} z}, \quad i=j=1,2,3, \ldots, 8,
$$

where the $c_{i}^{j}$ and $\kappa_{j}$ are the eigenvectors and eigenvalues, respectively, of the $8 \times 8$ matrix in (4.6) in the limit $z \rightarrow \infty$. The values of $\kappa_{j}$ are given by

$$
\begin{aligned}
& \kappa_{1,2}=\kappa_{5,6}=(1 / 2) \mp(1 / 2)\left(1+4 k^{2}\right)^{1 / 2}, \\
& \kappa_{3,4}=\kappa_{7,8}=-(1 / 2) \mp(1 / 2)\left(1+4 k^{2}\right)^{1 / 2} .
\end{aligned}
$$

Again, in order that all perturbations decay at $z \rightarrow \infty, C_{2}$ $=C_{4}=C_{6}=C_{8}=0$, which leaves the components of the final solution vector as

$$
\begin{aligned}
\phi_{i}\left(z, k_{x}, k_{y}, R\right)= & \sum_{j=1}^{8} C_{j}\left(k_{x}, k_{y}, R\right) \phi_{i}^{j}\left(z, k_{x}, k_{y}, R\right), \\
& i=1,3,5,7 .
\end{aligned}
$$

The wall boundary conditions are the same as those given in Sec. III A and allow the values of $C_{1,3,5,7}$ to be determined as nontrivial solutions of (3.8), i.e., for zeros of $\Delta\left(k_{x}, k_{y}, R\right)$ $=0$. Note that the differences between the solution of the linear stability analysis and the energetic analysis are that the $8 \times 8$ matrices in (3.4) and (4.6) differ and that here there is no $\omega$ dependence. As discussed in Sec. III A, in the linear stability analysis only the top left $4 \times 4$ block of the $8 \times 8$ matrix in (3.4) needs to solved if only the linear eigenvalues are required. However, here an equivalent reduction of (4.6) is only possible if $k_{y}=0$, otherwise the full eighth-order system must be solved. Indeed, it can be shown (see Sec. IV C) that the critical Reynolds number for energetic stability (below which there is no amplification of any disturbance) for a Hartmann layer with purely wall-normal imposed magnetic field is given by a two-dimensional disturbance with $k_{x}=0$, i.e., streamwise vortices, and not $k_{y}=0$.

\section{B. Non-normal magnetic field}

As with the linear stability analysis, formulation of the energetic stability problem when the imposed magnetic field is inclined in the $x$ or $y$ direction is similar to that discussed in Sec. III A with appropriately modified forms of (4.6) and (4.8). We have restricted the analyses by considering only the effects of $B_{x}$ or $B_{y}$ individually, and we use the same notation for the inclined fields as described in Sec. III B.

\section{Energetic stability results}

Figure 7 shows marginal curves for energetic stability for a selection of cases. For $k_{x}=0\left(\right.$ and $\left.k_{y} \neq 0\right)$ the curves are distinct for insulating and conducting walls but, as with the linear stability analysis, for $k_{y}=0$ (and $k_{x} \neq 0$ ) the insulating and conducting wall boundary conditions result in identical curves. Further, for $k_{x}=0$ the addition of a component of magnetic field in the $x$ direction has no effect on the energetic stability of the flow; similarly, for $k_{y}=0$ the addition of a component of magnetic field in the $y$ direction has no effect. However, for $k_{x}=0$ the addition of a component of magnetic field in the $y$ direction is stabilizing, as is the addition of a component of magnetic field in the $x$ direction for $k_{y}=0$.

Joseph ${ }^{14}$ gives a proof, following Busse, ${ }^{16}$ that for an odd velocity profile without electromagnetic forces (e.g., plane Couette flow) the critical Reynolds number for energetic stability is determined by considering only the cases

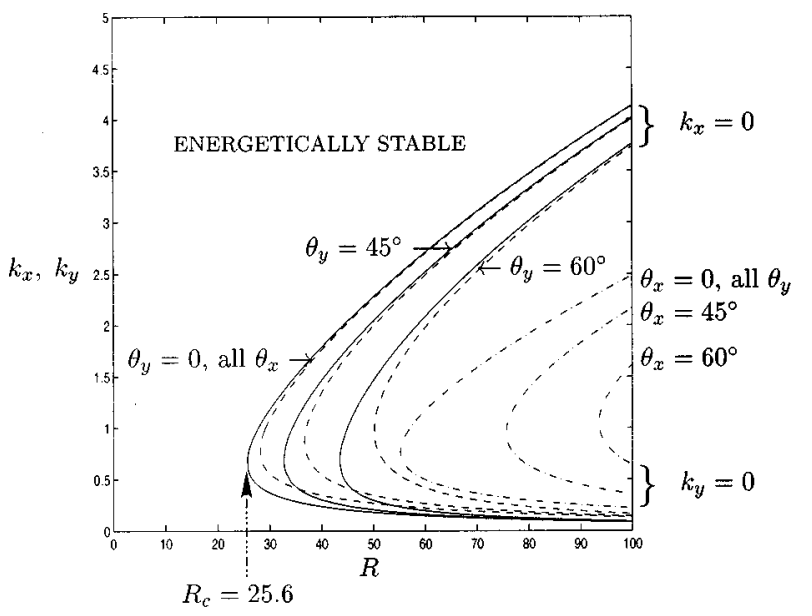

FIG. 7. Marginal curves for energetic stability: — insulating boundary; - - - conducting boundary; -.- both insulating and conducting boundary. 


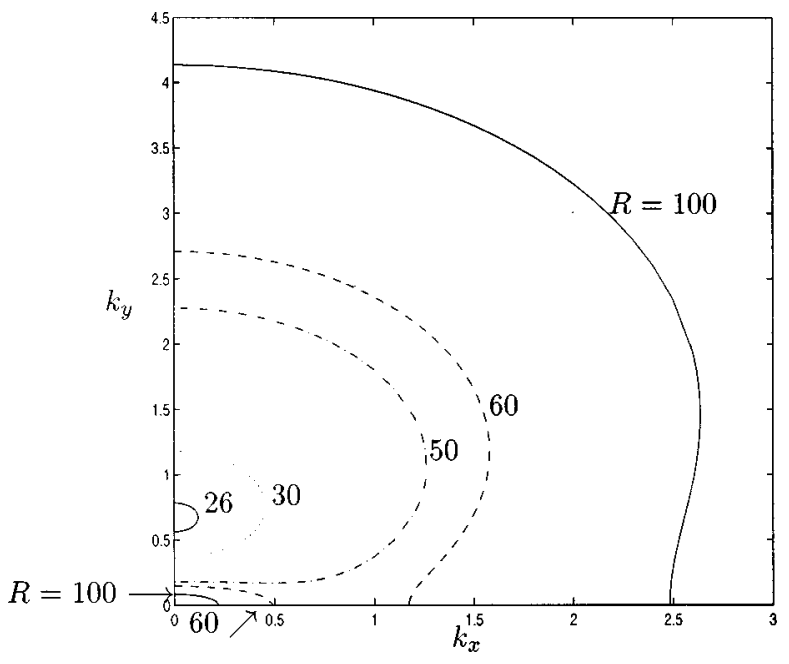

FIG. 8. Marginal curves for energetic stability for an insulating boundary and $B_{x}=B_{y}=0:-R=100 ;--R=60 ;-\cdot R=50 ; \cdots R=30 ;-R=26$.

$k_{x}=0$ or $k_{y}=0$. Figure 7 does not conclusively show whether these results also apply to the Hartmann layer. Although we can see that $k_{x}=0$ is more critical than $k_{y}=0$ for any (zero or nonzero) $B_{x}$, we do not know from Fig. 7 whether some combination of $k_{x} \neq 0$ and $k_{y} \neq 0$ would give a lower critical Reynolds number. Moreover, Fig. 7 suggests that for sufficiently large $B_{y}$ the marginal curve for $k_{y}=0$ becomes more critical than that for $k_{x}=0$, but again it may be that some combination of $k_{x} \neq 0$ and $k_{y} \neq 0$ is most critical. To clarify these issues we have calculated marginal curves for energetic stability in the $\left(k_{x}, k_{y}\right)$ plane for fixed values of $R$. Figure 8 shows the results for a purely normal imposed field. The points on the $k_{x}$ axis and $k_{y}$ axis are already given in Fig. 7. Here only the first quadrant is shown, but there is symmetry about both the $k_{x}$ axis and $k_{y}$ axis. The fact that the constant $R$ lines at the smaller values of $R$ are ellipses with a principle axis lying on the $k_{y}$ axis implies that as $R$ is reduced the "island" of instability does indeed shrink to the $k_{y}$ axis, i.e., $k_{x}=0$. Similarly, for $B_{x}=1$ and $B_{y}=1$ and an insulating boundary, Figs. 9 and 10, respectively, show that $k_{x}=0$ again gives the critical Reynolds number for energetic stability. However, for increased $B_{y}$, e.g., $B_{y}$ $\approx 2.75\left(\theta_{y}=70^{\circ}\right)$ in Fig. $11, k_{y}=0$ gives the critical Reynolds number. The results for a perfectly conducting pipe are qualitatively similar.

The energetic stability results for $B_{y} \neq 0$ (see Fig. 7) can be applied to the flow in a circular pipe with a magnetic field imposed in the $z$ direction i.e., normal to the axis of the pipe, which is in the $x$ direction. This field gives a local wallnormal component that varies as $\cos \theta_{y}$. At high Hartmann number layer that forms at the pipe wall approaches the Hartmann-layer solution for a flat plate. Around the circumference of the pipe, the thickness of the layer varies inversely with the local normal component of the magnetic field, i.e., as $\sec \theta_{y}$, and the local free-stream velocity varies as $\cos \theta_{y}$. Therefore, the local Reynolds number is constant. Figure 12 shows the variation in critical Reynolds number for energetic stability with $\theta_{y}$ for an insulating boundary. (The results for a perfectly conducting pipe are qualitatively similar.) Figure



FIG. 9. Marginal curves for energetic stability for an insulating boundary and $B_{x}=1$ and $B_{y}=0:-R=100 ;-\cdots R=80 ; \cdots R=60 ; \cdots R=30 ;-$ $R=26$.

11 shows that $\theta_{y}=0$ (and equally $180^{\circ}$ ) has the lowest critical Reynolds number $(\approx 25.6)$ and therefore the Hartmann layer on the $z$ axis is the first part of the layer around the pipe to become unstable to finite disturbances as the Reynolds number is increased. Beyond $\theta_{y} \approx 67^{\circ}$ the critical Reynolds number is approximately 55.2 and is given by $k_{y}=0$.

\section{DISCUSSION AND CONCLUSIONS}

We have considered the stability of an isolated Hartmann layer to both infinitesimal and finite perturbations. The linear stability results (i.e., for infinitesimal perturbations) were not entirely unexpected, given the approximate results of Lock ${ }^{3}$ and those of Takashima ${ }^{5,6}$ for both Poiseuille and Couette flows under a transverse magnetic field (both having a critical Reynolds number of 48311.016 at high Hartmann number). Here, we find that the critical Reynolds number for instability of the ideal Hartmann-layer solution with a wallnormal magnetic field is approximately 48250 (for both an

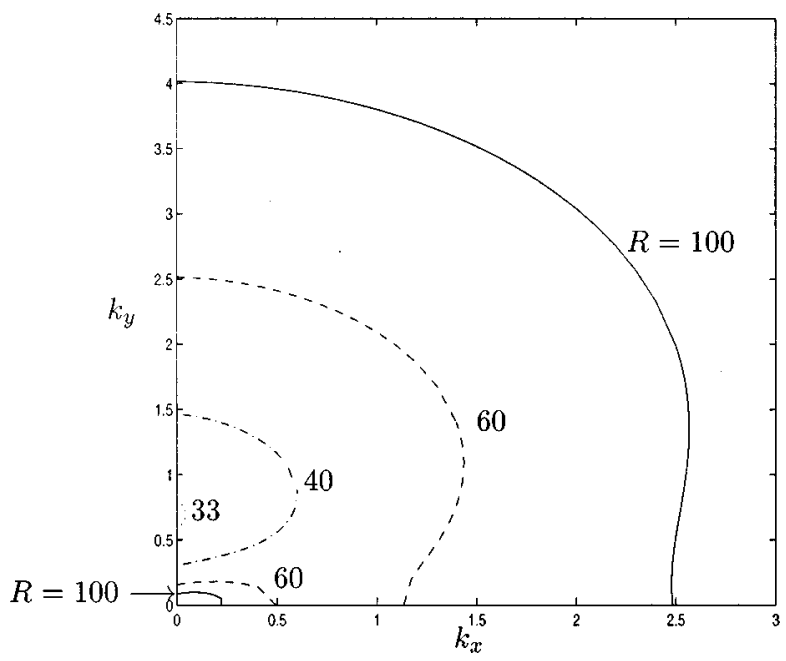

FIG. 10. Marginal curves for energetic stability for an insulating boundary and $B_{y}=1$ and $B_{x}=0:-R=100 ;--R=60 ;-\cdot R=40 ; \cdots R=33$. 


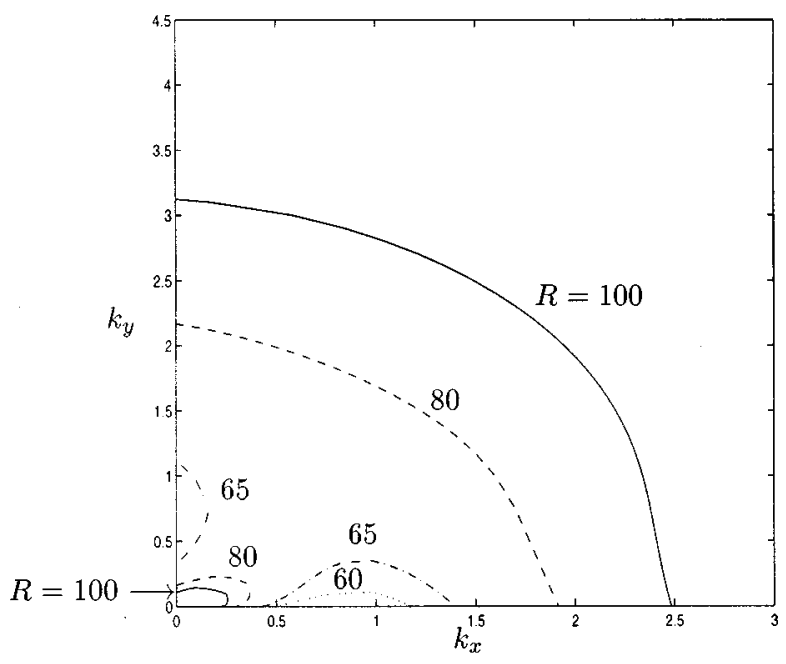

FIG. 11. Marginal curves for energetic stability for an insulating boundary and $B_{y} \approx 2.75$ and $B_{x}=0:-R=100 ;-\cdots R=80 ;-\cdot R=65 ; \cdots R=60$.

insulating and conducting boundary). The difference between our result and Takashima's is small (approximately $1.5 \%)$ and is probably due to differences in calculation method. We have also briefly considered the effects of inclining the magnetic field in the $x$ and $y$ directions. Despite the fact that Squire's theorem has not been proven in these cases, we restricted the analyses by considering only twodimensional perturbations with $k_{y}=0$. In this way, we found that inclination of the field in the $y$ direction resulted in the same marginal curve for linear stability as for a purely wallnormal field and that inclination in the $x$ direction has a small stabilizing effect. It may be that three-dimensional perturbations give a more critical Reynolds number for instability than two-dimensional perturbations when the magnetic field is inclined, but we feel that given that the critical values were so much larger than the observations of "transition" between turbulent and laminar flow in ducts at high Hartmann

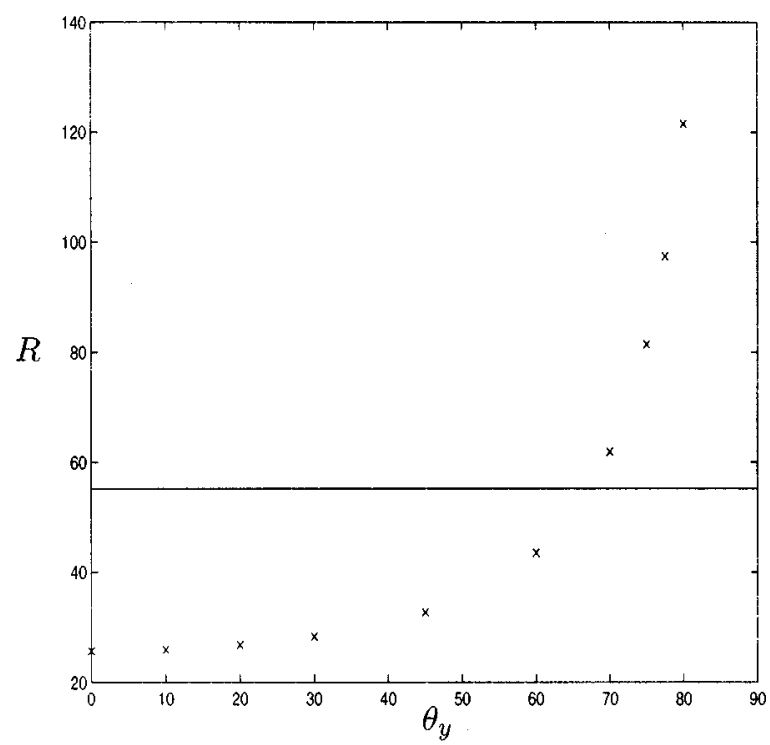

FIG. 12. Variation in critical Reynolds number for energetic stability with $\theta_{y}$ (in degrees) for an insulating boundary: $\times k_{x}=0 ;-k_{y}=0$. number that there is little insight to be gained from a linear stability analysis of three-dimensional perturbations. We believe that this discrepancy between early linear stability results and experimental observations of laminarization has led to the widespread feeling that the stability characteristics of the Hartmann layer have no bearing on the state (laminar or turbulent) of bounded MHD flows. However, our suggestion is that instability of the Hartmann layer does determine the state but that it is instability of finite, and not infinitesimal, perturbations that is fundamentally important. We base our belief that the state of a wide range of bounded (or partially bounded) MHD flows at high Hartmann number is due to the stability or instability of the Hartmann layers, rather than of the global flow, on the fact that experiments have shown that transition (in fact, laminarization) is controlled by the value of the ratio $\mathrm{Re} / \mathrm{Ha}$, rather than $\mathrm{Re}$ alone. If, as commonly believed, it were the global flow that controls this transition process, Re and Ha would be the two independent determining factors. Apart from the apparent irrelevance of duct cross section and aspect ratio, there is, unfortunately, no conclusive evidence to support our claim. (Even in the case of rectangular cavities with the long side parallel to the magnetic field, there are Hartmann layers along the short sides, defined by the same parameter $R$. For an infinite aspect ratio, the laminar flow would be Poiseuille flow unaffected by the magnetic field and linear stability would depend on Re only. This is not observed in experiments, probably because the aspect ratios are not sufficiently large. We claim that the Hartmann layers still govern the stability of all the finite aspect-ratio duct flows studied in experiments.) This is because experiments that have detected laminarization have largely done so through measurements of pressure drop along a section of ducted flow, rather than through detailed measurements that would determine the state, i.e., laminar or turbulent, of the Hartmann layer and global flow. There is, however, limited experimental evidence, reported by Branover, Gelfgat and Tsinober, ${ }^{17}$ that, for Reynolds numbers slightly higher than the threshold value, (electric potential) fluctuations are found only in the vicinity of the boundaries. Undoubtedly, there is a need for more detailed experiments to determine the state of the Hartmann layers.

Our energetic stability analysis for finite perturbations results in a critical Reynolds number of approximately 26 for a wall-normal field and shows the stabilizing effect of inclining the field in both the $x$ and $y$ directions. Although the critical Reynolds number is now much closer to experimental observed threshold values than that given by linear stability analysis, there is still an order of magnitude difference. This difference is, at least in part, due to the fact that the critical Reynolds number resulting from the energetic stability analysis must be viewed as a lower bound; the critical Reynolds number is the Reynolds number below which all (divergence-free) perturbation decay monotonically to zero and above which at least one perturbation does not decay monotonically. Monotonic decay of all disturbances is a very stringent condition, whereas allowing transient growth with ultimate decay is likely to result in a more realistic critical Reynolds number. Figure 13, which is adapted from Joseph, ${ }^{14}$ shows a schematic representation of the stability 
GLOBALLY \& MONOTONICALLY STABLE

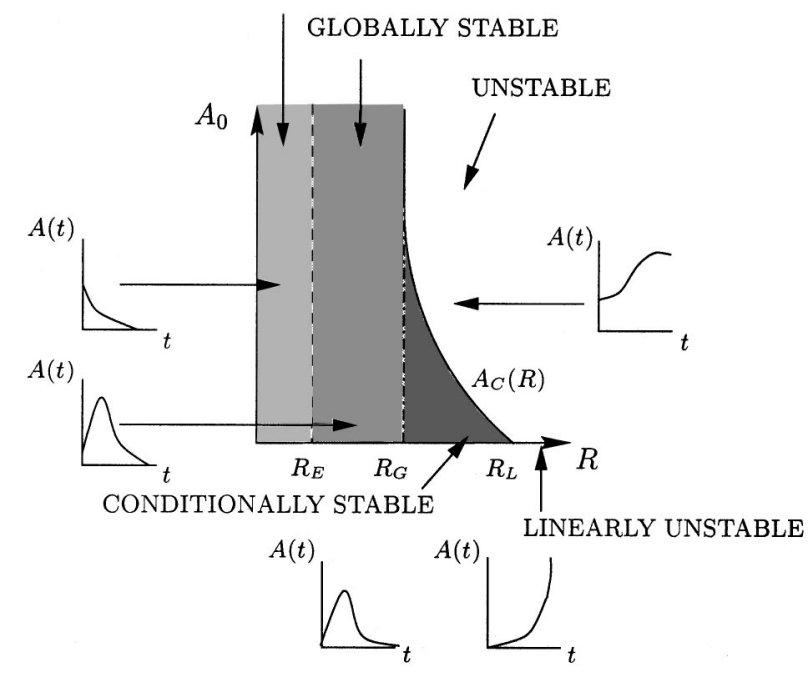

FIG. 13. Schematic representation of the stability bounds.

bounds, where $R_{E}$ is the critical Reynolds number derived from an energetic stability analysis, such as ours described in Sec. IV A, $R_{L}$ is the critical Reynolds number derived from a linear stability analysis, such as that described in Sec. III A, and $R_{G}$ is the Reynolds number below which all disturbances eventually decay. Here, $A(t)$ denotes the time-dependent amplitude of a perturbation and $A_{0}$ is the initial perturbation amplitude. Between $R_{E}$ and $R_{G}$ perturbations of infinitesimal or finite initial amplitude may grow transiently but they ultimately decay. This is also true below $A_{C}(R)$ between $R_{G}$ and $R_{L}$. We believe that the experimentally observed value for laminarization of $R \approx 200$ is a good approximation for $R_{G}$. To the right of $A_{C}(R)$, perturbations do not ultimately decay to zero, although the amplitude may saturate at some finite level. We have calculated $R_{E}$ as a lower bound approximation of $R_{G}$, which is likely to be a better indicator of the laminarization Reynolds number. Kreiss, Lundbladh, and Henningson ${ }^{18}$ have developed a method to calculate an estimate for $R_{G}$ (still a lower bound approximation, but better than $R_{E}$ ), however straightforward application of the procedure is precluded when the flow is semi-infinite, e.g., a boundary layer. Nevertheless, as for the Hartmann layer, the transition Reynolds number for plane Poiseuille flow and no magnetic field $(\approx 1000)$ is an order of magnitude greater than $R_{E}(\approx 50)$, which gives some support to our claim that instability of finite perturbation triggers transition. Further, Nagata ${ }^{19}$ has recently given interesting results from a nonlinear analysis on steady three-dimensional finite-amplitude solutions of plane Couette flow with a transverse magnetic field. He considers a range of Hartmann numbers, with the largest value being 10 for which he shows that the critical Reynolds number $\mathrm{Re}$ is about 3280 for the appearance of steady nonlinear solutions. This value of Hartmann number is not sufficiently large for the laminar Hartmann layers to be closely exponential, as considered here, but if we nevertheless consider these results, Nagata shows that steady threedimensional finite-amplitude disturbances exist for approximately $R>328$. This is in fair agreement with the experimental results for laminarization $(150<R<250)$. To make a real comparison, however, we would need to apply Nagata's analysis in the limit of high Hartmann number and consider unsteady, as well as steady, finite-amplitude solutions.

For high-Hartmann-number ducted flows, as well as Hartmann layers, there are parallel layers along boundaries parallel to the magnetic field. These parallel layers are thicker $\left(\delta^{*} / H^{*} \sim \mathrm{Ha}^{-1 / 2}\right)$ than the Hartmann layers $\left(\delta^{*} / H^{*} \sim \mathrm{Ha}^{-1}\right)$ and therefore for constant magnetic field intensity and free-stream velocity the Reynolds number based on the layer thickness is greater for the parallel layers than the Hartmann layers. This may suggest that the parallel layers are more unstable and therefore that they, not the Hartmann layers, control the "transition" process in ducted MHD flows. Although we could not find any reference to stability of parallel layers in the literature, it is true to say that parallel layers are stabilized by the friction due to the presence of Hartmann layers at their ends. Moreover, because the parallel-layer thickness is large, the contribution to the total wall shear stress from the parallel layers is negligible compared with that from the Hartmann layers. Whether the parallel layers are more or less unstable, it is therefore unlikely that laminarization (transition) of the parallel layers alone-assuming that with decreasing (increasing) Reynolds number it occurs before that of Hartmann layers - would be detected as a decreased (an increased) pressure drop along the duct, nor is it likely that laminarization (transition) of the parallel layers would globally change the flow. Furthermore, in circular-pipe flows under a magnetic field there are no parallel layers and these flows are observed experimentally to behave in the same way as flows in rectangular crosssection ducts, where there are parallel layers. The results of the energetic stability analysis (see Sec. IV C) favor the hypothesis that the Hartmann layers control "transition" because they show that the most unstable part of the circumferential layer is at zero inclination to the imposed field, i.e., where the field is purely normal to the wall, and the associated critical energetic Reynolds number (25.6) is the same as for the Hartmann layers in a duct with rectangular cross section, which is consistent with the experimentally observed similarity. In summary, we believe that the parallel layers play a passive role.

There are obvious similarities between the asymptoticsuction boundary layer and the Hartmann layer; both have exponential mean velocity profiles and both therefore have similarly high critical Reynolds numbers for instability of infinitesimal disturbances. (The shape of the exponential velocity profile is the dominant factor in determining the stability, not the electromagnetic damping of disturbances.) However, in the case of the asymptotic-suction boundary layer, experiments have shown the strongly stabilizing effect of introducing suction at the wall to a flat-plate hydrodynamical boundary layer; see Libby, Kaufmann, and Harrington. ${ }^{20}$ It seems that the laminar asymptotic-suction boundary layer conforms to the predictions from a linear stability analysis, provided the level of extraneous excitation from sources such as free-stream turbulence and wall roughness are small. This suggests that the same may be true for 
an initially laminar Hartmann layer in a low-disturbance environment, i.e., that it may be possible for the boundary layer to remain laminar up to the very large Reynolds numbers predicted by a linear analysis for instability. Thus far, experiments on high-Hartmann-number MHD flows have not investigated the transition from laminar to turbulent flow, instead they have considered the reverse process of laminarization. It is likely that there is a large degree of hysteresis between these processes and that the true transition Reynolds number in a "clean" environment is higher than the laminarization Reynolds number. ${ }^{9}$ This is simply because the finite-amplitude disturbances in the initially turbulent flow are unstable to much lower Reynolds numbers than infinitesimal disturbances and therefore the Reynolds number must be small to stabilize the Hartmann layer to the excitation of the finite-amplitude turbulent fluctuations. Clearly, despite the practical difficulties of creating the appropriate conditions, further experiments are needed in order to investigate the transition from laminar to turbulent flow of the Hartmann layer. It would also be interesting to compare the existing experimental results for laminarization of the Hartmann layer with laminarization of an initially turbulent asymptotic-suction boundary layer, in parallel with an energetic stability analysis.

\section{ACKNOWLEDGMENT}

R.J.L. acknowledges the support provided by a Dorothy Hodgkin Royal Society Fellowship.

${ }^{1}$ J. Hartmann and F. Lazarus, "Experimental investigations on the flow of mercury in a homogeneous magnetic field,' K. Dan. Vidensk. Selsk. Mat. Fys. Medd. 15(7), 1 (1937).

${ }^{2}$ S. Lundquist, "Studies in magneto-hydrodynamics,' Ark. Fys. 5, 297 (1952).
${ }^{3}$ R. C. Lock, "The stability of the flow of an electrically conducting fluid between parallel planes under a transverse magnetic field,' Proc. R. Soc. London, Ser. A 233, 105 (1955).

${ }^{4} \mathrm{P}$. H. Roberts, An Introduction to Magnetohydrodynamics (Longmans, Green, New York, 1967), Chap. 6.

${ }^{5} \mathrm{M}$. Takashima, "The stability of the modified plane Poiseuille flow in the presence of a transverse magnetic field," Fluid Dyn. Res. 17, 293 (1996).

${ }^{6} \mathrm{M}$. Takashima, "The stability of the modified plane Couette flow in the presence of a transverse magnetic field,' Fluid Dyn. Res. 22, 105 (1998).

${ }^{7} \mathrm{~W}$. Murgatroyd, "Experiments on magneto-hydrodynamic channel flow," Philos. Mag. 44, 1348 (1953).

${ }^{8}$ P. S. Lykoudis, "Transition from laminar to turbulent flow in magnetofluid mechanic channels," Rev. Mod. Phys. 32, 796 (1960).

${ }^{9}$ G. G. Branover, "Resistance of magnetohydrodynamic channels," Magnetohydrodynamics 3, 3 (1967).

${ }^{10}$ W. H. Press, S. A. Teukolsky, W. T. Vetterling, and B. P. Flannery, in Numerical Recipes, 2nd ed. (Cambridge University Press, Cambridge, 1992), p. 51.

${ }^{11}$ A. R. Wazzen, T. Okamura, and A. M. O. Smith, “The stability of water flow over heated and cooled flat plates," J. Heat Transfer 90, 109 (1968).

${ }^{12} \mathrm{H}$. B. Squire, "On the stability of three-dimensional distribution of viscous fluid between parallel walls,' Proc. R. Soc. London, Ser. A 142, 621 (1933).

${ }^{13}$ W. M'F. Orr, "The stability or instability of the steady motions of a liquid. II. A viscous liquid," Proc. R. Ir. Acad. Sect. A, Math. Astron. Phys. Sci. 27, 69 (1907).

${ }^{14}$ D. D. Joseph, Stability of Fluid Motions (Springer, New York, 1976), Vol. I, p. 182

${ }^{15}$ C. R. Doering and J. D. Gibbon, Applied Analysis of the Navier-Stokes Equations (Cambridge University Press, Cambridge, 1995), p. 29.

${ }^{16} \mathrm{~F}$. H. Busse, "A property of the energy stability limit for plane parallel shear flow,' Arch. Ration. Mech. Anal. 47, 28 (1972).

${ }^{17}$ G. G. Branover, Yu. M. Gelfgat, and A. B. Tsinober, "Turbulent magnetohydrodynamic flows in prismatic and cylindrical ducts," Magnetohydrodynamics 2, 3 (1966).

${ }^{18}$ G. Kreiss, A. Lundbladh, and D. S. Henningson, "Bounds for threshold amplitudes in subcritical shear flows," J. Fluid Mech. 270, 175 (1994).

${ }^{19}$ M. Nagata, "Nonlinear solutions of modified plane Couette flow in the presence of a transverse magnetic field," J. Fluid Mech. 307, 231 (1996).

${ }^{20}$ P. A. Libby, L. Kaufmann, and R. P. Harrington, “An experimental investigation of the isothermal laminar boundary layer on a porous flat plate," J. Appl. Spectrosc. 19, 127 (1952). 